\title{
Helical Ion Beams from Fluctuating Shock Structures
}

Urs Ganse ${ }^{* a, c, d}$, Rami Vainio ${ }^{b}$, Felix Spanier $^{c}$, Alex Ivascenko ${ }^{c}$, Minna Palmroth $^{d}$, Sebastian von Alfthan ${ }^{d}$, Yann Pfau-Kempf ${ }^{d}$

${ }^{a}$ Department of Physics, University of Helsinki,

${ }^{b}$ Department of Physics and Astronomy, University of Turku,

${ }^{c}$ Centre for Space Research, North-West University Potchefstroom,

${ }^{d}$ Finnish Meteorological Institute,

E-mail: urs.ganse@helsinki.fi

Using two separate simulation methods, kinetic-scale test-particle simulations and hybrid-Vlasov simulations, we have obtained evidence of nongyrotropic, helical beam structures in foreshocks of wavy shock structures. Artificial satellite observations with a simulated electrostatic analyzer modeled after the THEMIS ESA instrument show that in typical 2D cuts of velocity space, these structures are not properly visible. We instead propose a method to visualize the temporal development of the relevant part of phase space in order to obtain signatures of velocity-space spirals.

The 34th International Cosmic Ray Conference,

30 July- 6 August, 2015

The Hague, The Netherlands

\footnotetext{
* Speaker.
} 


\section{Introduction}

Interaction of charged particles with shocks in the heliosphere is one of the primary processes for creating solar energetic particles. The traditional assumption going into analytic models of particle shock interaction is adiabaticity and the conservation of adiabatic invariants, assuming the particle gyro radius to be much smaller than the shock width. The resulting accelerated particle beams would result in a clean loss-cone shaped particle distribution, with gyrotropic shape and no further substructure. This loss-cone distribution is typically used as the basis for models of particle propagation [1,2] and instabilities [3]. Both observations [4] and self-consistent plasma simulations [5], however, strongly indicate the presence of large-amplitude kinetic-scale structures in both the up- and downstream vicinity of a shock, making the adiabaticity assumption by itself highly questionable, and in-situ observation of particle beams upstream of shocks show a large variety of beam morphologies.

\section{Test-Particle Simulations}

Initially motivated to test shock-return rates and confirm analytic models of particle-shock interaction [6], a kinetic-scale test-particle system was developed to investigate the effect of different shock structures on the resulting beam distributions.

By taking the particle propagation system from the ACRONYM [7] particle-in-cell code, a thoroughly verified and validated codebase was used as the starting point for our modeling approach. It is using the common Boris-push [8] algorithm to propagate charged particle motion in a time-symmetric update process, using electric and magnetic field information as an input.

Unlike in a particle-in-cell code, in our model these fields are not solved self-consistently from simulation of Maxwell's equations, but given by an a-priori field model representing the shock structure of interest, with included kinetic-scale features.

\subsection{Setup}

The overall setup is that of a planar shock in a solar wind plasma flow, with field conditions far in the upstream and far in the downstream given according to the Rankine-Hugoniot conditions. Coordinate systems are chosen such that the $x$-axis forms the shock normal, with positive $x$ pointing towards the upstream direction, and the $y$ axis lying in-plane with the upstream magnetic field. The coordinates' origin is chosen such that $x=0$ is located in the centre of the shock transition. The shock is quantified by the parameters shock speed $v_{s}$, upstream flow speed $v_{w}$, compression ratio $R$, shock transition width $L$ and shock normal angle against the interplanetary magnetic field $\Theta_{B n}$, with a cross-shock potential being specified as fraction of the difference in kinetic energy across the shock.

In the examples presented here, we used a shock speed of $v_{s}=50 \mathrm{~km} / \mathrm{s}$, solar wind speed of $v_{w}=500 \mathrm{~km} / \mathrm{s}$, shock compression ratio $R=3.17$, shock normal angle $\Theta_{B n}=25^{\circ}$ and cross shock potential given by $12 \%$ of free kinetic energy, based on magnetospheric bow shock conditions.

The actual shock transition between upstream and downstream parameters is specified by choosing a shock profile function $f(x)$, which outlines the shape of the shock as an interpolation function between 0 and 1 . All simulation quantities, such as magnetic field strength, bulk plasma 
velocities and temperature, are then linearly interpolated between their up- and downstream values using expressions of the form

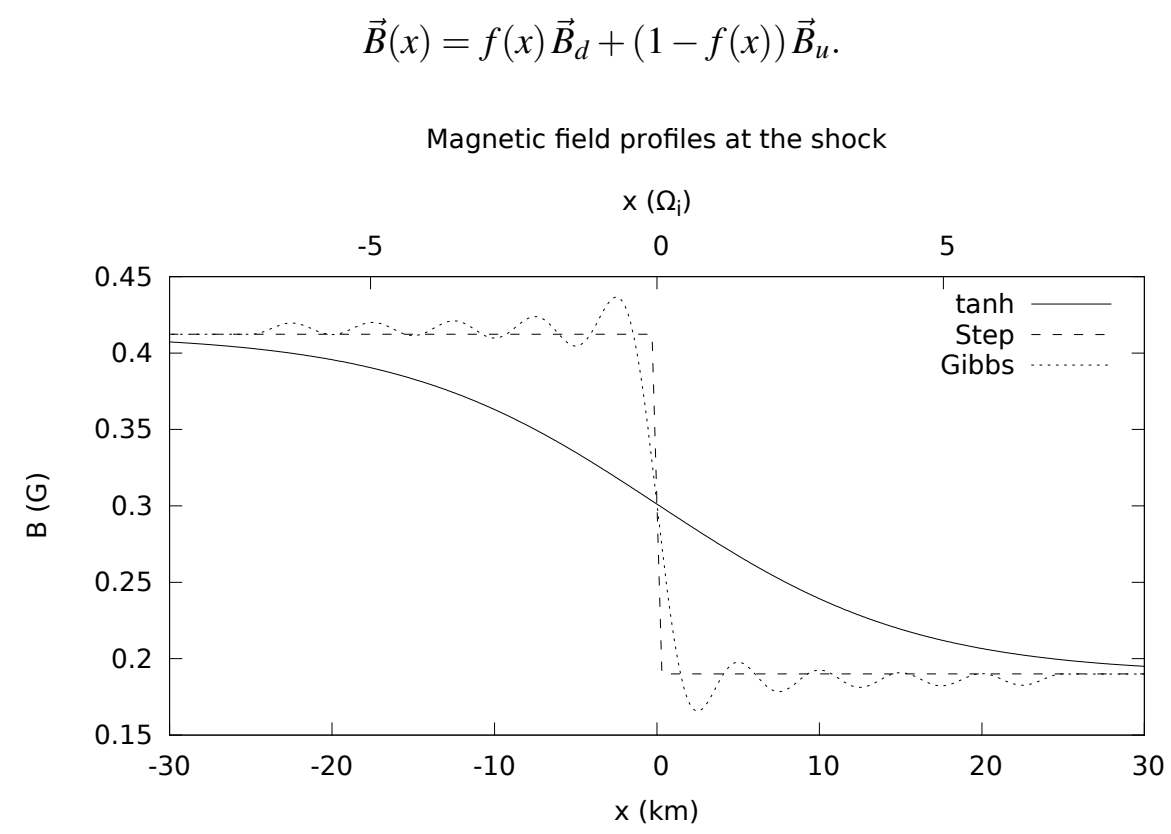

Figure 1: Example use of shock profile functions as employed in this study, giving different kinetic-scale structure to a shock's magnetic field transition.

Figure 1 shows examples of shock profile functions that can be employed in the code, including a smooth tanh- like transition, and a profile function obtained by using Gibb's phenomenon to have overoscillations both in front of and behind the central shock transition.

While the simulation runs with smooth shock transitions reproduce the expected loss-cone beam distributions, runs using the Gibb's phenomenon based shock profile with a wavy foreshock show a significant departure from the gyrotropic beam structure, with a clearly visible helical component in velocity space, as depicted on the left side of Figure 2.

\section{5D Global Vlasov Simulations}

To confirm that the helical beam structures are not just an artifact of the employed simulation method, comparison with results from 5D $(2 \mathrm{D}+3 \mathrm{~V})$ global Vlasov simulation runs using the Vlasiator [9] code was carried out.

As reported by [10], global simulations of the magnetospheric polar plane with radial IMF setups do likewise present a bow shock with kinetic-scale wave structures and shock reformation. Velocity space data from these runs show a wide variety of complex nongyrotropic beam morphologies in the Earth's foreshock, including a significant fraction of helix-shaped velocity space structures.

The right side of Figure 2 shows an example of a velocity space helix in the foreshock of Earth's magnetosphere, with very similar features to the ones obtained by the test particle simulation. 

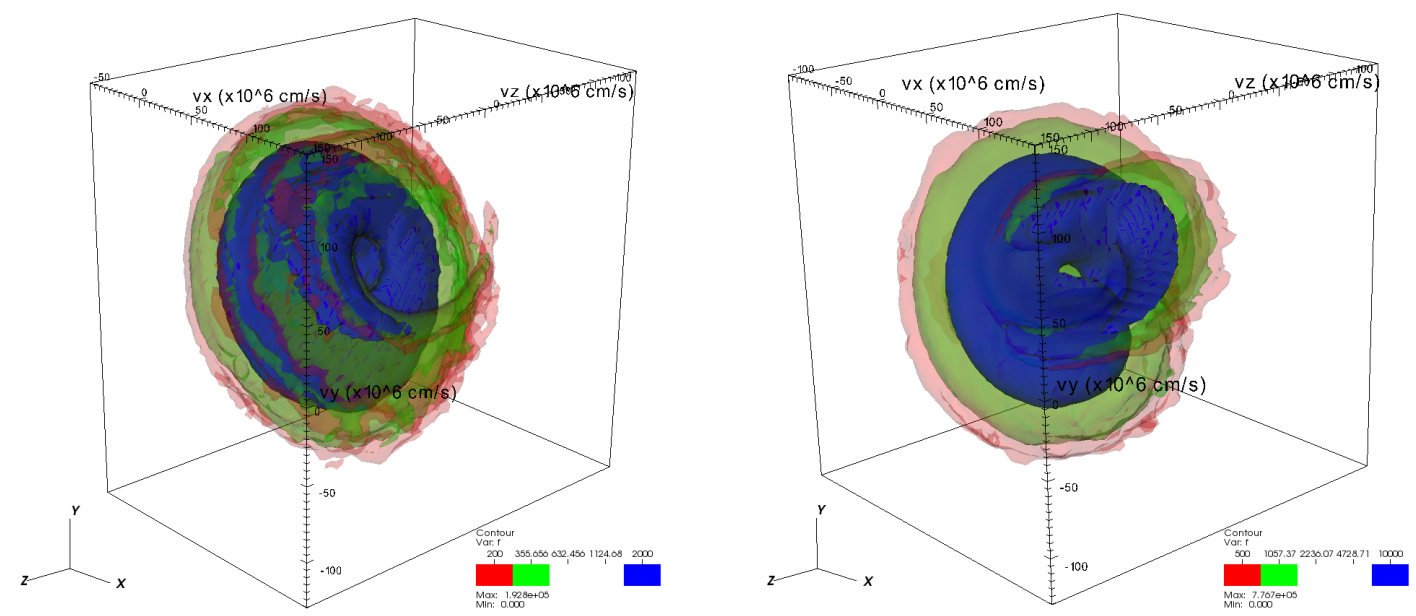

Figure 2: Three-dimensional foreshock velocity distributions. These examples of helix-shaped distribution functions obtained from kinetic-scale test-particle simulations (left) and hybrid-Vlasov simulations (right) of particle-shock interaction. The 3D axes give velocity space coordinates in $\mathrm{cm} / \mathrm{s}$, phase space density is in arbitrary units.

\section{Artificial Observations}

While helical beam structures are clearly visible in well-resolved simulation phase space outputs, characteristic signatures for them have not been visible in spacecraft measurements.

Based on the results presented above, artificial observations with realistic resolution of ion phase space densities were created. The process is based around simulating a virtual model of the ESA instrument's detector used in the THEMIS spacecraft [11], and the subsequent analysis steps of the THEMIS software suite.

To simulate the "top hat" electrostatic analyzer's angular field of view and energy resolution, each simulation particle (in the case of test-particle simulations) or phase space cell (in the case of Vlasov simulations) is transformed into the detector's observation plane, rejected if it is located outside of its view cone, and subsequently allocated to a detector bin. Figure 3 shows an example detector view in Earth's foreshock, from a Vlasiator simulation, showing both the bulk ion motion of incoming solar wind ions and the reflected beam population.

As in the actual spacecraft, the 32 such detector samples are taken at equal rotation angles of the virtual spacecraft around a freely chosen spin axis, and then reprocessed to create the following data products.

\subsection{D Plane Cuts}

A typical way of plotting the obtained 3D phase space data from electrostatic analyzers is to create $2 \mathrm{D}$ plane cuts, typically selecting a plane that contains the ambient magnetic field and/or bulk velocity.

Inherently 3D velocity space structures like the helix beams presented above however are difficult to image using these 2D cuts. Even in the best case, in which the helices center axis lies 


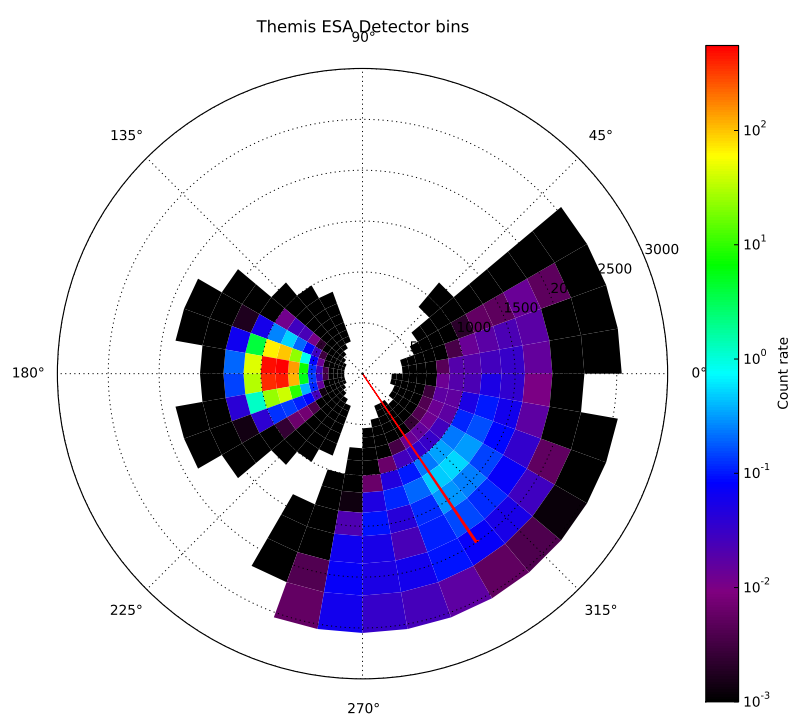

Figure 3: Example of a simulated spacecraft measurement showing the count rates of a virtual THEMIS ESA detector in the foreshock of Earth's bow shock, in a Vlasiator simulation. Both the thermal bulk population of incoming solar wind ions and the beam population are visible. The red arrow denotes the magnetic field direction.

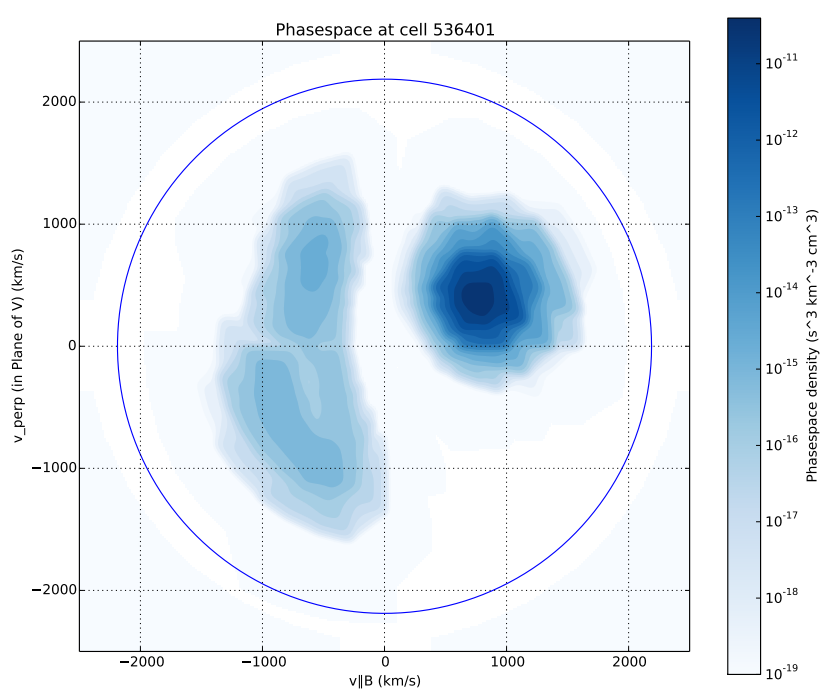

Figure 4: 2D plane cut created from synthetic THEMIS ESA observations, in the plane of magnetic field and bulk velocity vector. The velocity-space helix is not discernible in this type of plot. 
precisely in the cut plane, the resulting distribution is basically indistinguishable from a gyrotropic ring. Figure 4 shows $2 \mathrm{D}$ plane plots created by this method from the distribution functions depicted on the right side of Figure 2, in which the $x$-axis is given by the velocity component parallel to the local magnetic field direction, and the $y$-axis by the perpendicular component in the plane that contains the bulk velocity.

The helical nature of the distribution function in these types of plots only becomes evident when investigating their temporal evolution as animations or image sequences, which is unfortunately not very practical for publication. Instead, we propose a different way of plotting the temporal evolution of certain selected directions of phase space most relevant to the spiral shape.

\subsection{Directions-on-a-Cone Plots}

While the assumption of a loss-cone shaped beam is not valid in the presence of a velocityspace helix, the loss cone angle

$$
\sin ^{2} \phi=\frac{B_{u}}{B_{d}}
$$

still serves as a reasonable estimate of the helices' directional offset against the magnetic field

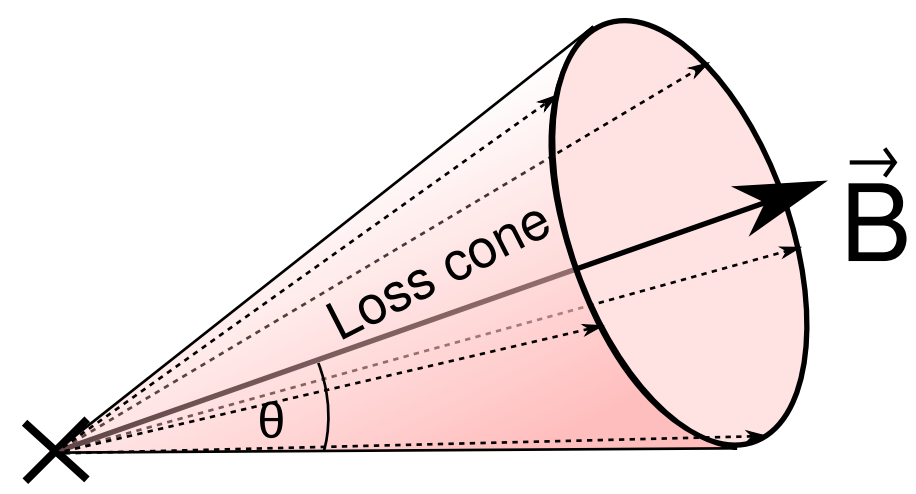

Figure 5: Distribution of sampling directions in order to measure velocity space helices. The samples, depicted as dashed arrows, are arranged on the surface of a cone with the nominal loss cone angle $\theta$ around the magnetic field vector.

direction. One-dimensional cuts of velocity space on the surface of the loss cone tend to sample the helix reasonably well, and easily allow temporal development to be plotted. Figure 5 illustrates the distribution of sampling directions.

The resulting patterns in temporal evolution of phase space densities in these directions are shown in Figure 6. As the velocity-space helices rotate due to gyration, similar structures show up in successive bins, with decreasing energy according to the spiral winding. If similar signatures were to be found in observational data, this would be indicative of the presence of helix-shaped beams.

\subsection{Conclusions}

Using two separate simulation methods, kinetic-scale test-particle simulations and hybridVlasov simulations, we have obtained evidence of nongyrotropic, helical beam structures in foreshocks of wavy shock structures. Artificial satellite observations with a simulation of the ESA 


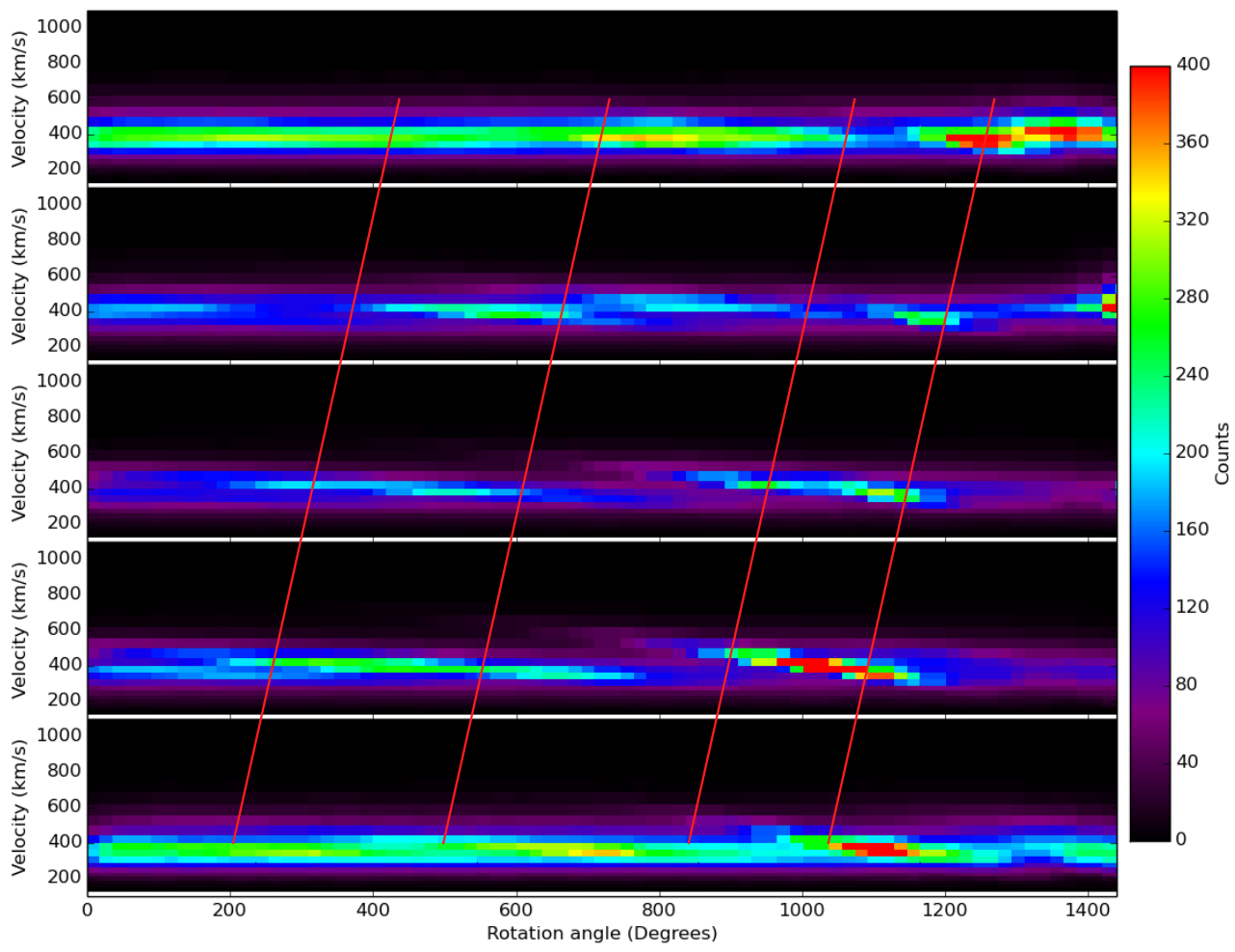

Figure 6: Signatures of helical beam structures in the temporal evolution measured along a finite number of velocity directions on the surface of the loss cone. As the spiral rotates due to gyration, similar signatures with decreasing energy can subsequently be seen is successive bins. The red lines highlight some of these features.

detector onboard THEMIS show that in typical 2D cuts of velocity space, these structures are not properly visible. We instead proposed a method to visualize the temporal development of the relevant part of phase space in order to obtain signatures of velocity-space spirals.

\section{Acknowledgements}

UG acknowledges financial support by the German Research Foundation (DFG) under grant agreement GA 1968/1.

We thank Heli Hietala for insightful comments about the 2D plane plots and THEMIS ESA analysis.

\section{References}

[1] R. Vainio, A. Pönni, M. Battarbee, H. E. J. Koskinen, A. Afanasiev, and T. Laitinen, A semi-analytical foreshock model for energetic storm particle events inside 1 AU, Journal of Space Weather and Space Climate 4 (Feb., 2014) A8. 
[2] S. Lange, F. Spanier, M. Battarbee, R. Vainio, and T. Laitinen, Particle scattering in turbulent plasmas with amplified wave modes, Astronomy \& Astrophysics 553 (May, 2013) A129.

[3] U. Ganse, P. Kilian, F. Spanier, and R. Vainio, Nonlinear wave interactions as emission process of type ii radio bursts, The Astrophysical Journal 751 (June, 2012) 145(6pp).

[4] R. Vainio, E. Valtonen, B. Heber, O. E. Malandraki, A. Papaioannou, K.-L. Klein, A. Afanasiev, N. Agueda, H. Aurass, M. Battarbee, S. Braune, W. Dröge, U. Ganse, C. Hamadache,

D. Heynderickx, K. Huttunen-Heikinmaa, J. Kiener, P. Kilian, A. Kopp, A. Kouloumvakos, S. Maisala, A. Mishev, R. Miteva, A. Nindos, T. Oittinen, O. Raukunen, E. Riihonen, R. Rodríguez-Gasén, O. Saloniemi, B. Sanahuja, R. Scherer, F. Spanier, V. Tatischeff, K. Tziotziou, I. G. Usoskin, and N. Vilmer, The first sepserver event catalogue 68-mev solar proton events observed at 1 au in 1996-2010, J. Space Weather Space Clim. 3 (2013) A12.

[5] P. Savoini, B. Lembege, and J. Stienlet, On the origin of the quasi-perpendicular ion foreshock: Full-particle simulations, Journal of Geophysical Research: Space Physics 118 (2013), no. 3 1132-1145.

[6] Battarbee, M., Vainio, R., Laitinen, T., and Hietala, H., Injection of thermal and suprathermal seed particles into coronal shocks of varying obliquity, A\&A 558 (2013) A110.

[7] P. Kilian, T. Burkart, and F. Spanier, The influence of the mass ratio on particle acceleration by the filamentation instability, in High Performance Computing in Science and Engineering '11 (W. E. Nagel, D. B. Kröner, and M. M. Resch, eds.), pp. 5-13. Springer, Berlin Heidelberg, 2012.

[8] J. P. Boris, Relativistic plasma simulation-optimization of a hybrid code, Proceeding of Fourth Conference on Numerical Simulations of Plasmas (November, 1970).

[9] S. von Alfthan, D. Pokhotelov, Y. Kempf, S. Hoilijoki, I. Honkonen, A. Sandroos, and M. Palmroth, Vlasiator: First global hybrid-vlasov simulations of earth's foreshock and magnetosheath, Journal of Atmospheric and Solar-Terrestrial Physics 120 (2014), no. 024 - 35.

[10] Y. Kempf, D. Pokhotelov, O. Gutynska, L. B. Wilson III, B. M. Walsh, S. v. Alfthan, O. Hannuksela, D. G. Sibeck, and M. Palmroth, Ion distributions in the earth's foreshock: Hybrid-vlasov simulation and themis observations, Journal of Geophysical Research: Space Physics 120 (2015), no. 5 3684-3701.

[11] J. McFadden, C. Carlson, D. Larson, M. Ludlam, R. Abiad, B. Elliott, P. Turin, M. Marckwordt, and $\mathrm{V}$. Angelopoulos, The themis esa plasma instrument and in-flight calibration, Space Science Reviews 141 (2008), no. 1-4 277-302. 